Service social

\title{
L'expérience des jeunes autochtones pris en charge par la protection de la jeunesse : entre déracinement et émancipation
}

\section{Marie-Hélène Gagnon-Dion, Jacinthe Rivard et Céline Bellot}

Volume 64, numéro 1, 2018

URI : https://id.erudit.org/iderudit/1055892ar

DOI : https://doi.org/10.7202/1055892ar

Aller au sommaire du numéro

Éditeur(s)

École de service social de l’Université Laval

ISSN

1708-1734 (numérique)

Découvrir la revue

Citer cet article

Gagnon-Dion, M.-H., Rivard, J. \& Bellot, C. (2018). L'expérience des jeunes autochtones pris en charge par la protection de la jeunesse : entre déracinement et émancipation. Service social, 64(1), 79-102. https://doi.org/10.7202/1055892ar
Résumé de l'article

Cet article vise à partager le point de vue de jeunes autochtones quant à leur vécu de prise en charge par la protection de la jeunesse et aux effets de cette prise en charge. L'article s'appuie sur les données recueillies dans le cadre d'une recherche plus large sur la judiciarisation et la défense des droits des personnes en situation de pauvreté. Les résultats font ressortir trois types d'expérience de prise en charge impliquant un placement des jeunes autochtones : les résistants qui vivent le placement comme un déracinement les résilients qui vivent le placement comme une émancipation ; les résignés qui vivent le placement comme un détachement. Ces résultats montrent que les liens des jeunes autochtones avec leur milieu d'origine pendant le placement influencent grandement leur façon de vivre la prise en charge par la protection de la jeunesse, les effets de cette prise en charge ainsi que le regard qu'ils portent sur cette expérience. L'article propose des pistes d'intervention visant à améliorer le bien-être des jeunes autochtones pris en charge par la protection de la jeunesse.
Tous droits réservés (C) Service social,
Ce document est protégé par la loi sur le droit d'auteur. L'utilisation des services d’Érudit (y compris la reproduction) est assujettie à sa politique d'utilisation que vous pouvez consulter en ligne.

https://apropos.erudit.org/fr/usagers/politique-dutilisation/ 


\title{
L'expérience des jeunes autochtones pris en charge par la protection de la jeunesse : entre déracinement et émancipation
}

\author{
GAGNON-DION, Marie-Hélène, M. Sc., doctorante en travail social \\ Consultante pour les services sociaux autochtones et chargée de cours \\ Université de Montréal \\ RIVARD, Jacinthe, Ph.D., professeure associée, coordonnatrice de recherche \\ Chercheure associée à la Chaire de recherche du Canada sur l'Évaluation des actions \\ publiques à l'égard des jeunes et des populations vulnérables (CREVAJ) \\ Université de Montréal \\ BELLOT, Céline, Ph.D., directrice de l'École de travail social et professeure titulaire, \\ Directrice de l'Observatoire sur les profilages racial, social et politique \\ Université de Montréal
}

\section{RÉSUMÉ}

Cet article vise à partager le point de vue de jeunes autochtones quant à leur vécu de prise en charge par la protection de la jeunesse et aux effets de cette prise en charge. L'article s'appuie sur les données recueillies dans le cadre d'une recherche plus large sur la judiciarisation et la défense des droits des personnes en situation de pauvreté. Les résultats font ressortir trois types d'expérience de prise en charge impliquant un placement des jeunes autochtones : les résistants qui vivent le placement comme un déracinement; les résilients qui vivent le placement comme une émancipation ; les résignés qui vivent le placement comme un détachement. Ces résultats montrent que les liens des jeunes autochtones avec leur milieu d'origine pendant le placement influencent grandement leur façon de vivre la prise en charge par la protection de la jeunesse, les effets de cette prise en charge ainsi que le regard qu'ils portent sur cette expérience. L'article propose des pistes d'intervention visant à améliorer le bienêtre des jeunes autochtones pris en charge par la protection de la jeunesse.

Mots-clés : protection de la jeunesse, jeunes autochtones, prise en charge, placement, interventions sociales, récits de vie, sociologie de l'expérience, typologie.

\begin{abstract}
This article aims to share the point of view of young Aboriginals about their experience of youth protection and the effects of this care. The article draws on data collected as part of broader research on the judiciarization and advocacy of people living in poverty. The results highlight three types of care experience involving placement of Aboriginal youth: "Resistants » who experience placement as uprooting; "Resilients » who live the placement as an emancipation; the "Resigned » who live the placement as a detachment. These results show that the relationship of Aboriginal youth with their home environment during placement greatly influences how they experience youth care, the effects of
\end{abstract}


caregiving and the way they look at it. This article proposes ways to improve the well-being of Aboriginal youth in care of youth protection.

Keywords: youth protection, indigenous youth, care, placement, social interventions, life stories, sociology of experience, typology. 
La surreprésentation des jeunes autochtones dans les systèmes de protection de l'enfance au Canada fait écho à un passé marqué par la colonisation et les politiques assimilatrices. Avant la colonisation, les familles autochtones s'occupaient de leurs enfants selon leurs pratiques culturelles et leurs traditions. Traditionnellement, dans l'organisation familiale autochtone, les membres de la famille élargie partageaient les responsabilités en matière de soins et d'entraide, et les systèmes de clans étendaient encore davantage les réseaux d'obligations mutuelles (Castellano, 2002). Les enfants étaient vus comme sacrés et, puisqu'ils étaient sacrés et vulnérables, leur protection, qui prenait différentes formes, était une responsabilité collective (Castellano, 2002 ; Little Bear, 2000). Avec l'arrivée des colons européens, ces structures sociales et familiales ont été bouleversées, ceux-ci ayant imposé leurs propres visions du monde et leurs modes de fonctionnement. Les modèles traditionnels de soins ont alors été remplacés par des pratiques étatiques qui prirent d'abord la forme des pensionnats indiens, où l'on séparait les enfants de leur famille durant toute l'année scolaire afin de les préserver de l'influence de leur milieu familial (Commission de vérité et réconciliation du Canada [CVR], 2015 ; Blackstock, 2009 ; Commission royale sur les peuples autochtones [CRPA], 1996 ; Kline, 1992). À partir de 1960, les régimes provinciaux de protection de l'enfance s'appliquèrent aux populations autochtones avec l'objectif d'offrir les mêmes services aux populations autochtones qu'au reste de la population canadienne, dans une perspective d'égalité, de neutralité et d'universalité (Kline, 1992). Toutefois, pendant une vingtaine d'années, parce qu'on disait vouloir donner la chance aux enfants de grandir dans des foyers plus avantagés, des milliers d'enfants autochtones ont été adoptés, la majorité du temps par des familles blanches, ce qui a valu le nom de «sixties scoop 》 ou « rafle des années 60 » à cette période historique (McKenzie et al., 2016 ; Blackstock, 2009 ; Fournier et Crey, 1997). Les pratiques de l'époque visaient aussi à briser les liens de l'enfant avec sa famille en le séparant de sa fratrie et en l'éloignant de son milieu d'origine (Fournier et Crey, 1997 ; Kline, 1992).

Les pensionnats et la rafle des années 1960 ont eu un impact majeur sur l'identité de générations entières en séparant des milliers d'enfants de leur famille, de leur communauté, de leur culture. Les jeunes autochtones qui ont fréquenté les pensionnats ou qui ont été élevés dans des familles blanches pendant la rafle des années 1960 ont grandi avec une connaissance et une compréhension limitées de leurs racines, ce qui a engendré chez eux d'importants problèmes d'identité et provoqué des répercussions négatives dans leur vie (Bennett et Blackstock, 2002 ; Sinclair, 2007 ; Carrière, 2008). Beaucoup de ces jeunes se sont trouvés dans une situation où ils n'avaient pas accès à leur héritage culturel tout en ayant de la difficulté à s'identifier à la culture « d'accueil » (Bennett, Blackstock et De La Ronde, 2005). Le fait d'être coincés entre deux cultures engendrait chez ces jeunes une image négative d'eux-mêmes qui les poussait souvent à se tourner vers la consommation d'alcool, de drogues ou de solvants, pour s'évader ou faire face au stress (Bennett et Blackstock, 2002). Les conséquences de ces problèmes d'identité pouvaient s'accentuer à l'adolescence et au début de l'âge adulte et se traduire par des idées suicidaires et une plus grande tendance à commettre des crimes (Teichroeb, 1997).

Aujourd'hui, les autorités provinciales chargées de la protection de l'enfance reconnaissent généralement les impacts qu'ont eus les politiques et les pratiques du passé sur les enfants autochtones, affirment leur volonté de maintenir les enfants dans leur milieu familial et se veulent plus sensibles à la réalité autochtone. Au Québec, la Loi sur la protection de la jeunesse (LPJ) mentionne que l'intervention de la Direction de la protection de la jeunesse (DPJ) doit être adaptée aux caractéristiques des communautés autochtones et le gouvernement peut aussi conclure avec une 
nation autochtone une entente établissant un régime particulier de protection, pour autant que celle-ci soit conforme aux principes généraux de la LPJ (Demers, 2009). Cependant, malgré cette plus grande sensibilité de la part des autorités provinciales, au Québec, les enfants autochtones sont cinq fois plus susceptibles de faire l'objet d'un placement que les enfants non autochtones (Sinha et al., 2011). L'histoire se répète-t-elle ? Quelle est l'expérience des jeunes autochtones relativement à la prise en charge dans le contexte actuel de la protection de la jeunesse au Québec?

Des chercheurs canadiens ont tenté de faire la lumière sur la surreprésentation des jeunes autochtones en protection de la jeunesse. La plupart s'entendent pour dire que ce sont des facteurs structurels, principalement la pauvreté et les risques qui en découlent, qui expliquent en grande partie cette surreprésentation (Blackstock, Trocmé et Bennett, 2004 ; Sinha et al., 2011; Trocmé, Knoke et Blackstock, 2004 ; Tourigny et al., 2007). La colonisation et les politiques d'assimilation sont pointées du doigt car elles ont détérioré les conditions de vie des populations autochtones et entraîné, par le fait même, ces facteurs de risque (Blackstock et al., 2004 ; Blackstock, 2011 ; Trocmé et al., 2004 ; Tourigny et al., 2007). En outre, encore aujourd'hui, les familles autochtones ont accès à moins de ressources relatives au bien-être de leurs enfants que le reste de la population canadienne (Blackstock et Trocmé, 2005) et ces iniquités touchent particulièrement les « mesures moins perturbatrices », c'est-à-dire celles qui permettent de garder les enfants autochtones dans leur famille (Blackstock, 2011). Certains mettent en avant aussi les conséquences intergénérationnelles des pensionnats indiens comme l'une des causes de la surreprésentation des enfants autochtones en protection de la jeunesse (Dion Stout et Kipling, 2003 ; Bennett et al., 2005 ; Castellano, Archibald et DeGagné, 2008 ; CRPA, 1996). En enlevant aux parents la responsabilité de leurs enfants, le régime des pensionnats a privé ces derniers d'un modèle parental, ce qui a eu des conséquences dévastatrices sur les compétences relatives à l'éducation des enfants, compétences qui sont transmises d'une génération à l'autre. En somme, la littérature met en lumière le fait que les familles autochtones sont aux prises avec des problèmes sociaux plus importants que l'ensemble des Canadiens et des Québécois, mais avec moins de ressources pour y répondre.

Si ces travaux mettent en exergue des causes et des explications à cette surreprésentation, nous n'avons recensé au Canada aucune étude qui interroge le point de vue des jeunes sur leur réalité et leur expérience de prise en charge. Puisqu'au regard de la Loi sur la protection de la jeunesse, toute décision doit être prise en fonction de ce qui correspond au plus grand intérêt de l'enfant, le point de vue des jeunes autochtones est primordial si on veut que les interventions répondent à cet objectif. De plus, les services de protection de l'enfance n'ayant pas toujours su répondre au bien-être des jeunes autochtones, il importe de connaître le sens que ces jeunes donnent aujourd'hui à l'intervention de la protection de la jeunesse dans leur vie.

Cet article s'appuie sur les données d'une recherche sur la judiciarisation et la défense des droits des personnes en situation de pauvreté, dont un volet s'est tenu à Val-d'Or, au Québec, auprès de jeunes autochtones résidant en milieu urbain qui ont vécu une prise en charge par la protection de la jeunesse. En donnant une voix à ces jeunes, nous désirons faire entendre leur point de vue quant à l'intervention de la protection de la jeunesse dans leur vie et, par le fait même, intégrer leurs discours dans les discussions concernant la protection de l'enfance autochtone. Dans le cadre de cette recherche, nous avons déjà fait ressortir que les jeunes autochtones rencontrés considèrent l'intervention de la DPJ 
comme une mesure de protection qui leur a permis de se soustraire à des conditions de vie difficiles (Gagnon Dion, Rivard et Bellot, 2017). Le présent article vise à présenter la perspective de ces jeunes sur le vécu et les effets de la prise en charge et à faire ressortir les significations qu'ils donnent à cette expérience. Nous inspirant de l'analyse typologique de Schnapper (2005), nous présenterons dans cet article trois figures types de l'expérience de la prise en charge rapportée par les jeunes autochtones : les résistants, les résilients et les résignés. Nous discuterons ensuite des enjeux que soulèvent ces résultats pour l'intervention dans le but d'améliorer les pratiques auprès des jeunes autochtones en protection de la jeunesse et le bien-être de ces jeunes.

\section{CADRe THÉORIQUe ET MÉthodologiQue}

Cette étude s'inspire de la sociologie de l'expérience. Le cadre théorique proposé par Dubet (1994) conçoit les individus comme des êtres autonomes dans la construction de leur histoire de vie, tout en considérant l'influence des conditions sociales dans lesquelles ces individus évoluent. Cette conception théorique, qui fait partie du grand courant de la sociologie compréhensive, ne considère pas les individus comme étant passifs devant les conditions sociales, mais plutôt comme des acteurs qui construisent eux-mêmes leur rapport au monde. Ce rapport au monde se dessine cependant à l'intérieur d'un cadre social qui en limite, dans une certaine mesure, les potentialités.

Dans le champ théorique de la sociologie de l'expérience, l'expérience sociale des individus résulte de l'articulation de trois logiques d'action : 1) l'intégration sociale ; 2) la stratégie ; et 3) la subjectivation. C'est par l'analyse de ces trois logiques d'action qu'il est possible d'expliquer les réalités individuelles telles que les acteurs les comprennent et, par le fait même, les réalités sociales. Selon la logique de l'intégration sociale, l'acteur se définit par les appartenances qu'il vise à maintenir ou à renforcer au sein de la société. L'analyse de l'intégration sociale explore la subjectivité des acteurs et leur rapport « culturel » et « identitaire » au monde. La deuxième logique d'action, «la stratégie », renvoie aux manières dont les acteurs vont tirer parti des situations dans lesquelles ils se trouvent. L'analyse de la stratégie passe donc également par la subjectivité des acteurs mais s'intéresse à leur rapport "d'intérêts » au monde. La troisième logique d'action, «la subjectivation », résulte des tensions produites par les deux autres logiques d'action, soit l'intégration sociale et la stratégie. Si ces dernières apparaissent à l'acteur comme des réalités du monde, la subjectivation est plutôt une mise à distance de celles-ci par l'acteur. Ainsi, l'analyse de la subjectivation se penche sur la subjectivité de l'acteur pour faire émerger son rapport «critique » au monde. En somme, la subjectivité des acteurs permet de comprendre comment ceux-ci construisent leur réalité dans les conditions de vie qui sont les leurs, tout autant qu'elle nous permet de comprendre les systèmes sociaux à travers la façon dont les acteurs les comprennent. Dans le cas de cette étude, la sociologie de l'expérience offre un cadre théorique où l'on part du point de vue des jeunes autochtones pour comprendre leurs réalités individuelles de prise en charge, ce qui permet par le fait même de cerner les enjeux de la rencontre entre les Autochtones et le système québécois de protection de l'enfance.

Les données et témoignages utilisés dans cet article proviennent d'une étude plus vaste portant sur la judiciarisation des personnes en situation de pauvreté ${ }^{1}$, dont un volet s'est tenu à Val-d'Or auprès de

\footnotetext{
${ }^{1}$ Recherche partenariale intitulée' " Judiciarisation et défense des droits des personnes en situation de pauvreté ", financée par le FQRSC, programme Actions concertées, et conduite par Céline Bellot.
} 
jeunes autochtones. Des entretiens non directifs, de type récit de vie (Bertaux, 2010), ont été réalisés auprès de huit jeunes autochtones, âgés de 18 à 24 ans, quatre femmes et quatre hommes, résidant en milieu urbain et ayant vécu une prise en charge par la protection de la jeunesse. Le recrutement s'est fait par l'entremise d'organismes de Val-d'Or intervenant auprès de jeunes autochtones, dont le Centre d'amitié autochtone de Val-d'Or, et par le Centre jeunesse de l'Abitibi-Témiscamingue. C'est lors d'une consultation de ces organismes que s'est établi le choix de recueillir les récits de jeunes résidant en milieu urbain puisque, d'une part, les jeunes des communautés autochtones de la région de l'Abitibi sont très sollicités par les chercheurs et que, d'autre part, on en sait très peu sur les jeunes autochtones qui font le choix d'habiter en ville ou qui en sont natifs. Les participants de cette étude sont nés soit dans une communauté autochtone, soit en milieu urbain, mais ils ont en commun le fait d'avoir choisi de s'établir en ville à l'atteinte de leur majorité.

Cette recherche a été approuvée par le Comité d'éthique de la recherche de la Faculté des arts et des sciences de l'Université de Montréal (CERFAS). La participation aux entretiens s'est réalisée sur une base volontaire et les jeunes ont donné leur consentement libre et éclairé. Les entretiens visaient à faire émerger le discours des participants autour de dimensions liées à la prise en charge telles que les conditions de vie entourant la prise en charge, les acteurs présents ou absents durant l'enfance, la nature de la prise en charge, la compréhension et la perception de cette prise en charge, les interactions avec les différents intervenants et les conditions de vie actuelles. Le déroulement de l'entretien visait à recueillir le discours des participants autour des thèmes explorés, sans en imposer le cours ${ }^{2}$ (Daunais, 1995). Les entretiens ont été enregistrés et transcrits mot à mot sur support informatique. Une analyse thématique, puis typologique inspirée de Schnapper (2005), a permis de faire ressortir différents types d'expérience des jeunes autochtones par rapport à leur parcours de prise en charge. Pour ce faire, il s'agissait d'abord de déconstruire les récits des jeunes en unités de sens, puis de regrouper celles-ci autour des dimensions de la prise en charge explorées par les entretiens. De nouvelles dimensions ont aussi été créées lorsque des éléments étaient récurrents et significatifs dans le discours des jeunes. C'est par exemple le cas de la dimension "Les acteurs présents ou absents durant la prise en charge ". Ces dimensions ont ensuite été analysées en articulant les liens entre les pensées, les émotions et les actions des jeunes. À ce stade, nous avions un portrait très détaillé, mais compartimenté, de l'expérience des jeunes. L'étape de reconstruction des données a été réalisée par une analyse typologique : des types ont été formés à partir des ressemblances, et ce, pour chacune des logiques d'action proposées par Dubet $(1994)^{3}$, d'abord par rapport au vécu des jeunes lié à la prise en charge, puis aux effets de la prise en charge dans leur vie. Trois groupes sont apparus sous chacun de ces deux thèmes, avec les mêmes jeunes à l'intérieur de ces groupes. Une analyse croisée, toujours à partir des logiques d'action de Dubet (1994), a permis de préciser trois types d'expérience.

\section{RÉSULTATS}

Nous tenons d'abord à souligner la générosité des jeunes rencontrés dans le cadre de cette étude qui ont bien voulu partager le récit de leur expérience de prise en charge. Ces jeunes nous ont paru très ouverts et même reconnaissants de pouvoir raconter librement leur parcours de vie et de faire entendre

\footnotetext{
${ }^{2}$ La volubilité des jeunes rencontrés étant limitée, nous avons fait appel à des techniques de reformulation et de reflet visant à faciliter le discours.

${ }^{3}$ Tel qu'il a été mentionné, ces logiques d'action sont l'intégration sociale, la stratégie et la subjectivation.
} 
leur point de vue sur leur prise en charge. Ils nous ont inspirées par leur compréhension claire de leur expérience de vie et nous ont ébranlées par la charge de leurs émotions, qu'elles soient positives ou négatives. Ces résultats se veulent les plus fidèles possible à leurs témoignages.

L'analyse des témoignages des jeunes autochtones rencontrés relativement à leur expérience de prise en charge par la protection de la jeunesse fait émerger trois figures types : les résistants (trois participants), les résilients (trois participants) et les résignés (deux participants). II faut d'emblée mentionner que cette prise en charge a amené tous les jeunes de notre échantillon à vivre un ou plusieurs placements. II ressort des résultats que les relations avec le milieu d'origine pendant le placement représentent l'élément qui a le plus marqué le parcours des jeunes dans cette prise en charge. Tous les jeunes rencontrés ont mis un accent particulier sur leurs relations avec les membres de leur famille immédiate et élargie. D'autres éléments, tels que l'âge des jeunes lors de la prise en charge, la durée de la prise en charge ou la trajectoire de placement, apparaissent, dans le discours des jeunes, non significatifs du point de vue de leur influence sur le vécu de la prise en charge et sur les effets que celle-ci a eus sur eux. D'une part, les jeunes y ont accordé peu d'importance dans leurs témoignages et, d'autre part, on retrouve dans les trois figures types des jeunes d'âges différents, des trajectoires de placement plus ou moins longues ainsi que des nombres de placements variés. Ainsi, un jeune ayant vécu de nombreux placements et un autre n'en ayant vécu qu'un seul peuvent percevoir tous deux que l'intervention de la protection de la jeunesse a eu un impact bénéfique dans leur vie. En revanche, la proximité avec la famille immédiate et élargie pendant le placement apparaît influencer la façon dont ils ont vécu la prise en charge, les effets de celle-ci sur eux et leur perception de l'intervention de la protection de la jeunesse.

\section{Les résistants : des jeunes qui se perçoivent comme entre deux mondes}

«On dirait que ce n’était pas ma place. »

\section{Le placement : une distance avec le milieu d'origine}

Le parcours de prise en charge des résistants est marqué par l'absence de liens significatifs avec la famille d'origine durant la prise en charge. S'ils ont voulu quitter les conditions de vie difficiles dans lesquelles ils se trouvaient dans leur milieu familial, ces jeunes veulent quand même rester en lien avec leur famille, et particulièrement avec leur mère pendant le placement. Les jeunes appartenant à la figure des résistants tentent le plus possible de garder une proximité avec leur mère en voulant la voir fréquemment, mais ils sont confrontés à un désengagement de la part de celle-ci. Leur parcours de prise en charge est ponctué de déceptions à cet égard :

Mère (désengagement)

Elle me mentait tout le temps. Elle disait tout le temps qu'elle allait être là pour moi et qu'elle allait m'appeler, mais elle ne m'appelait jamais, elle ne venait jamais me voir. Ça c'était 
quand j'étais en famille d'accueil. [... $]^{4}$ Je lui avais déjà dit [ce que je vivais], mais elle n'a rien voulu savoir. Elle me dit qu'elle m'aime, qu'elle va tout faire, mais non.

Je la voyais, mais je ne la voyais pas comme je voulais donc j'ai décidé d'abandonner ça, de passer à autre chose et de me concentrer sur quelque chose d'autre. Elle disait qu'elle venait me voir, mais elle ne venait pas me voir. Elle me faisait des promesses en l'air et elle ne les tenait jamais, donc un moment donné, je me suis tanné et j'ai passé à autre chose. [...] Je commençais à en avoir plein mon casque de me faire jouer dans le dos comme ça. J'ai catché vite.

En ce qui a trait au père, les jeunes de la figure des résistants présentent une relation non significative causée soit par son absence durant leur enfance, ce qui provoque une distance relationnelle difficilement récupérable, soit par son désengagement durant la prise en charge. II faut noter que cela est également vrai pour tous les jeunes rencontrés.

\section{Père (absence relationnelle)}

Mon père habitait ici. Je l'ai connu quand je suis arrivé à [nom de la ville]. Sinon avant je ne le connaissais pas. [...] Je le voyais rarement. Quand je le voyais, j'y parlais pas beaucoup. J'ai été quelques fois chez eux, mais je ne restais pas là longtemps. Je ne le connaissais pas... [...] Je restais là même pas cinq minutes.

\section{Père (désengagement)}

Mon père, il était à [nom d'une communauté autochtone], il ne vient jamais ici. [...] II m'appelle des fois, mais il ne vient jamais nous voir. Avant, je pleurais tout le temps quand mon père ne venait pas, mais je me suis habituée. [...] [J'aurais aimé ça le voir plus souvent] mais il n'était jamais là.

Les jeunes de la figure des résistants ont aussi tous en commun l'absence de liens continus avec leur famille élargie pendant leur parcours de placement. La famille élargie a une grande importance pour eux - «Maman n'était pas là, j'avais besoin de voir une de mes tantes » -, mais celle-ci a été effacée de leur vie à un moment ou l'autre de leur parcours de prise en charge.

\section{L'effet de la prise en charge : un déracinement}

Le vide créé par la distance avec le milieu d'origine provoque une tension identitaire chez ces jeunes. Cette tension identitaire est visible dans leurs discours par la division qu'ils font entre le " monde làbas », celui des communautés qui correspond à leurs origines, à « [leurs] racines », et le « monde des Blancs ", celui de la ville qui correspond au milieu d'accueil. Ainsi, même s'ils considèrent tous qu'ils ont de très bonnes ou d'excellentes familles d'accueil parce que celles-ci leur offrent de meilleures conditions de vie et parce qu'ils y développent des liens affectifs importants, ils ont de la difficulté à accepter ce nouveau milieu de vie. Se sentant perdus entre deux mondes et considérant que leur

\footnotetext{
${ }^{4}$ Par souci de concision et pour mettre l'accent sur le discours des jeunes, les parenthèses remplacent les reflets et
} reformulations de l'intervieweuse. 
« place » devrait être " quand même » auprès de leur mère et de leur communauté, les résistants ont de la difficulté à s'adapter aux situations qui surviennent dans leur parcours de prise en charge.

[Ma sœur] aussi elle a vécu plein d'affaires. Je pense que c'était pire que moi. Je ne sais pas, c'est tout plus pire. [...] [Je parle de violence], oui. D'abus sexuel. C'est pour ça que je voulais partir de [la communauté autochtone]. En même temps, je me sentais bien parce que c'était là mes racines.

J'essaie de me tenir à l'écart parce que je ne me sens pas chez nous [dans la famille adoptive], on pourrait dire. Pendant un moment, je me suis senti chez nous, mais plus les idées passaient, plus je me rendais compte que ce n'était pas ma place.

Les fugues, les tentatives de suicide et les conflits avec la famille d'accueil représentent leur façon de résister à leur prise en charge, d'exprimer leur mal-être et leur volonté d'un « meilleur ailleurs ». C'est le cas d'une jeune qui exprime un «besoin » d'être en lien avec ses « racines » et qui fuguait sans cesse de ses lieux de placement pour retourner dans son milieu d'origine. C'est aussi le cas d'un autre jeune qui se disait très proche de sa mère d'accueil qu'il identifiait « comme [sa] mère ", mais qui était toutefois incapable de se sentir bien dans son milieu, ce qui s'est traduit par de nombreuses fugues et tentatives de suicide :

Quand j'avais 12-13 ans, j'ai commencé à fuguer. J'ai commencé à fuguer parce que je n'étais pas bien. [...] Je n'arrêtais pas de fuguer. À 16 ans, la dernière fois que j'ai fugué, j'ai fait une tentative de suicide. J'étais tanné. J'avais fugué à 15 ans, j'avais fait une tentative de suicide après et ils [les parents d'accueil] m'ont repris. Après ça, j'avais été en thérapie. Ils m'ont repris quand j'avais fini ma thérapie. Après ça j'ai re-fugué, j'ai rechuté et j'ai recommencé à boire... Ouais, j'ai re-fugué et j'avais fait encore une tentative de suicide. Ma famille d'accueil ne m'a plus repris parce qu'ils avaient peur que je le refasse encore. J'ai beaucoup pleuré. [...] Oui [j'aurais aimé ça qu'ils me reprennent]. J'ai dit « laisse-moi une chance » puis j'avais dit autre chose, mais ils ne pouvaient pas, ils pleuraient.

Ces jeunes qui vivent leur placement comme un déracinement dessinent un parcours de prise en charge rempli d'obstacles. Par exemple, pendant leur prise en charge, tous les jeunes de cette figure affirment avoir été victimes d'intimidation de la part de leurs pairs, puisqu'en se trouvant entre « deux mondes », ils se distinguent à la fois des jeunes du milieu d'accueil par leurs origines et du milieu d'origine parce qu'ils sont imprégnés des particularités du milieu d'accueil :

\section{Intimidation (dans le milieu d'accueil)}

Dès les premiers jours où je suis arrivé [en ville], j'allais jouer dehors et je faisais face à l'intimidation. Je n'en parlais pas parce que dans ma culture, mettons que tu es à [nom d'une communauté autochtone] et qu'il se passe de quoi, il ne faut pas que tu en parles sinon la prochaine fois ça va se passer plus mal. Donc là, je n'en parlais pas parce que je ne veux pas que ça empire. Je n'en ai jamais vraiment parlé. Ma mère [d'accueil] ne savait pas vraiment à quoi je faisais face à l'école et je n'en ai jamais vraiment parlé non plus. [...] 
J'inventais tout le temps une histoire à chaque bleu que je me faisais faire, à chaque marque que j'avais, je m'inventais de quoi pour ne pas foutre la merde.

\section{Intimidation (dans le milieu d'origine)}

[...] après ma fugue, ma première fugue. J'étais à [nom d'une communauté autochtone]. Les filles là-bas avant avaient tendance à mettre des gros gilets avec des jeans ordinaires. Une fois, j'étais arrivée là-bas avec, tu sais des jeans style déchiré ? Avec une camisole quand même assez ajustée, mais qui était jolie. C'était la première fois que je buvais et on m'a battue après. J'étais toute seule. J'étais toute seule contre trois. On disait que j'avais osé embrasser le chum à une de mes amies qui était là, ce n'était pas vrai. [...] Des filles m'avaient battue. Il y avait une [Blanche - exprimé dans sa langue d'origine].

Les interactions avec les intervenants sont aussi généralement synonymes d'embûches pour ces jeunes et ils ont souvent l'impression de ne pas être écoutés ou compris par leurs intervenants, particulièrement en ce qui a trait aux décisions qui les concernent.

À chaque fois que je demandais quelque chose, ils disaient tout le temps non. Ils ne voulaient jamais que je sorte. [...] Des fois, aujourd'hui, je demande à ma T.S. si je peux ravoir ma petite sœur un peu. Des fois je ne le demande pas à elle, je le demande à sa famille d'accueil parce qu'elle ne veut jamais.

En somme, l'expérience de prise en charge de ces jeunes, qui implique autant les événements avant la prise en charge que pendant celle-ci, a eu l'effet de les fragiliser et ils semblent, même après leur majorité, vivre toujours les répercussions de ce parcours :

II n'y a pas longtemps, je trouve ça encore dur, j'ai tombé enceinte et je ne voulais pas... Je voulais le garder mais en même temps je ne voulais pas être comme ma mère, je ne voulais pas suivre les traces de ma mère, je ne sais pas si tu comprends... Je n'étais pas vraiment prête. Je trouve ça dur encore, je n'arrête pas de faire des cauchemars. [...] Des fois je pleure dans la nuit... quand je fais des cauchemars, je vois des bébés, je commence à pleurer et c'est dur.

II y a des fois que j'aimerais re-consommer mais si je fais ça, je vais rechuter et je ne veux pas. Je ne veux pas que ma fille vive la même chose que moi. Elle a besoin de nous. [...] Justement je pensais à ça, c'est à cause de la consommation que mes parents ils m'ont perdue. Puis je ne voulais pas faire ça. Je ne voulais pas faire la roue.

\section{La synthèse de la figure des Résistants : une protection « partielle »}

Le regard posé par les jeunes appartenant à la figure des résistants sur la prise en charge montre que le placement à l'extérieur de leur famille les protège contre des conditions de vie difficiles mais que cela a l'effet d'un déracinement car leur besoin d'appartenance à leur milieu d'origine n'est pas satisfait. Puisqu'ils ne sont pas capables de se sentir bien dans leur nouveau milieu, d'y sentir leur affiliation, le placement à l'extérieur de leur famille représente pour eux une protection « partielle » : 
Je trouve que c'est bien [le placement], mais... Je trouve que c'est une bonne chose, parce que ça permet aux gens qui sont en détresse d'avoir une meilleure famille, d'avoir une meilleure qualité de vie. D'avoir une meilleure vie. II faut juste savoir l'apprécier. [...] Je sais que, eux autres, qui vivent dans les réserves, ce n'est pas la meilleure des vies. C'est très pauvre là-bas et il y a souvent beaucoup de violence. Il y a de la violence comme dans les familles et quand il se passe de quoi, il faut que tu te taises parce que si tu t'ouvres ta bouche, la prochaine fois ça va se passer plus dangereusement. C'est plus bad pour toi. [...] En même temps, même si je suis là-bas ou chez des Blancs, c'est revenu à la même chose parce que, oui là-bas, j'aurais été battu physiquement, mais vivre chez des Blancs tu es battu psychologiquement parce que, à chaque jour, tu te fais traiter de noms, de différent, parce que justement ils te perçoivent différent parce que tu as l'air différent. Donc tu as l'air méchant ou tu n'es pas bien.

\section{Les résilients : des jeunes qui retirent le meilleur des deux mondes «Je ne changerais rien. »}

\section{Le placement : en proximité avec la famille immédiate}

Le parcours de prise en charge des résilients se démarque par la présence de contacts fréquents et significatifs avec leur famille immédiate, leur mère et leur fratrie, et aussi parfois avec des membres de leur famille élargie. En ce qui concerne la relation avec leur mère, les résilients sont proches de celleci pendant la prise en charge, soit parce qu'ils la voient de façon régulière et constante, soit parce qu'ils la voient fréquemment et vivent une grande complicité avec elle. Pendant la prise en charge, les jeunes de la figure des résilients font souvent face aux mêmes comportements de leur mère que ceux qui ont amené leur placement, mais cela n'affecte pas la fréquence des visites ou la qualité de leur relation. Les résilients ne manifestent pas de rancune vis-à-vis de leur mère et ils lui pardonnent autant les comportements qui ont motivé leur prise en charge que ses comportements pendant la prise en charge. Les contacts fréquents avec leurs frères et sœurs durant leur prise en charge sont également très significatifs pour eux. De plus, lorsque la famille élargie est présente pendant la prise en charge, celleci joue un rôle important de soutien.

\section{Mère et fratrie}

Oui [je voyais encore ma mère], mais pas souvent. Je dirais deux fins de semaine par mois. [...] J'aimais les sorties. J'aimais pas mal ça retourner chez ma famille. [...] À chaque fois que je revenais à la maison, c'était naturel. C'est sûr que j'avais plus de... je respectais plus les règles à la maison, le fait d'avoir été habituée en famille d'accueil.

Je le voyais partir des fois [mon père], il allait marcher sur le grand chemin. C'est là que j'en profitais pour aller voir mes petits frères. Faire de gros câlins à mes petits frères. Je parlais avec ma mère. C'était le seul temps que je pouvais parler avec ma mère. [...] Avec ma mère j'avais une bonne relation. [...] Même aujourd'hui encore. [...] Tabarnak, on parle quand moi et ma mère on se parle. Quand on parle, on peut parler pendant deux heures nous autres. Deux heures, une heure. Ma blonde des fois elle écoute, elle écoute tout le temps. « Vous 
avez toujours quelque chose à dire vous autres ! »qu'elle dit tout le temps ma blonde. «Ben quoi, c'est ma mère! », je lui dis.

Le premier mois que j'étais là toute seule, mes autres sœurs étaient chez mon père. Elles ont laissé tomber chez mon père et sont venues dans une famille d'accueil. Je n'étais plus là toute seule, j'étais avec ma grande sœur. [...] Je commençais à m'ennuyer là toute seule, pendant un mois.

\section{Famille élargie}

[...] Après [que mon père m'a mis dehors de la maison] j'ai été rester chez ma tante [nom de la tante]. La sœur à ma mère, sa petite sœur. Je suis resté là-bas pour, je dirais, trois mois.

\section{L'effet de la prise en charge : une émancipation}

La continuité des contacts avec la mère, la fratrie et parfois la famille élargie constitue pour les jeunes de la figure des résilients un ancrage identitaire. Les jeunes de cette figure parlent de leur " chez nous », de leur «famille » ou de leur «communauté » quand ils se réfèrent à leur milieu d'origine ; ils parlent de « là-bas », de « chez eux » ou « des Blancs » lorsqu'ils se réfèrent au milieu d'accueil. Ce discours identitaire n'est toutefois pas traversé par une tension entre les deux mondes. Les résilients côtoient deux mondes différents, mais ces mondes ne se trouvent pas en contradiction : ils cohabitent de façon harmonieuse en répondant à des besoins différents. Pour ces jeunes, la présence essentielle des membres de leur famille immédiate durant le placement comble leur besoin d'appartenance, tandis que leurs parents d'accueil, ou plus rarement leurs intervenants, occupent une place primordiale dans leur vie en répondant à leurs besoins de sécurité matérielle et affective. Par le fait même, les jeunes de cette figure sont ouverts et réceptifs vis-à-vis du milieu d'accueil et retirent le meilleur de chacun des milieux de vie dans lesquels ils évoluent, tel que le montre les témoignages suivants:

\section{Famille immédiate}

Moi je suis une personne qui est vraiment attachée à sa famille. Peu importe ce qui arrive, je vais tout le temps les aimer.

\section{Famille d'accueil}

[La personne dans ma vie qui a été la plus marquante], c'est [nom de la $2^{\mathrm{e}}$ mère d'accueil], celle qui m'a aidée avec mon fils. Elle a vraiment eu un gros impact, sur moi et avec mon fils. [...] On se disait vraiment les vraies affaires. Et elle m'a vraiment aidée avec mon loyer et tout ça. C'est elle qui nous a emménagés, elle et son mari, elle nous a vraiment aidés, elle nous a donné du stock. Je suis bien contente pour ça... C'est vraiment des bonnes personnes. [...] Ils nous ont beaucoup aidés.

Les jeunes de la figure des résilients s'arrangent pour que les choses se passent bien durant leur parcours de prise en charge. Par exemple, dans leurs interactions avec leurs intervenants, ils font en sorte de pouvoir retirer le maximum de leur situation : 
Pour que les services sociaux agissent correct avec nous autres, il faut qu'on soit correct avec eux autres. Parce que, j'ai entendu du monde, à force de grandir quand j'étais en famille d'accueil : «Elle est bien pute ma T.S., elle est bien chienne ma T.S. », des affaires de même. «Elle ne me laisse pas sortir. " «Qu'est-ce que tu fais ? », je disais. « J'ai fugué ! » «Ben voilà la raison pour laquelle tu n'as pas le droit », je disais. "Va donc chier », chaque personne me disait ça. Une semaine plus tard, les personnes s'excusaient parce qu'elles voyaient que j'avais raison parce que moi je sortais chaque semaine.

Les jeunes de cette figure ne perçoivent pas les événements qui se présentent dans leur vie comme des obstacles, mais plutôt comme des occasions pour grandir. Ces jeunes considèrent leur prise en charge comme une possibilité d'améliorer leurs conditions de vie et jugent que leur vie devient plus « facile » à la suite de leur placement par la protection de la jeunesse. En outre, chez les résilients, on constate que l'expérience de vie dans un milieu d'accueil leur permet de cheminer sur le plan personnel et de s'affranchir des difficultés personnelles vécues avant et pendant leur placement:

Parce que [mon beau-père] savait que j'avais changé depuis que je suis en famille d'accueil. [...] C'est que j'étais rendu moins naïf, j'étais plus... J'avais moins peur, donc lui il ne sait jamais quand il va le faire [acte de violence] si je vais appeler la police. Ou s'il faisait quelque chose à ma mère. [...] Avant, moi j'étais bien naïf. Je ne disais rien parce que j'avais peur. Maintenant, je n'ai plus peur de rien.

Avec [nom de la $1^{\text {ère }}$ mère d'accueil], la première année, on commençait à se parler, donc je me confiais à elle après. C'est ça qui m'a rendue plus ouverte. Être capable de m'exprimer. J'avais vraiment de la difficulté à m'exprimer avant. J'étais plus renfermée. [...] Parce que les familles d'accueil où j'ai été m'ont vraiment aidée. [...] Sur ma personne, moimême. En dedans. M'exprimer et tout ça. Aller chercher des ressources, des ressources d'aide.

\section{La synthèse de la figure des Résilients : une protection « totale »}

Pour les résilients, le placement à l'extérieur du milieu d'origine représente le mieux qu'il pouvait leur arriver puisqu'ils vivent cette situation comme une émancipation. La présence des membres de leur famille immédiate durant leur placement, combinée à celle des membres de la famille d'accueil, leur permet de se libérer des difficultés personnelles liées à leur passé familial. Les résilients se représentent donc leur prise en charge comme une protection « totale » :

Être placée, c'était vraiment le mieux. Je vois ça de même maintenant. [...] Oui [j'ai l'impression que ç'a été les meilleurs choix qui ont été faits], parce que là je suis vraiment contente. De ma vie. Où je suis rendue. [...] Moi je pourrais dire que ce n'est pas là pour rien. Ce n'est pas là pour rien et ça peut vraiment aider. Au début, tu trouves ça un petit peu louche, mais quand tu arrives à la fin, c'est sûr que ça a fait un impact.

Le vécu à l'extérieur de [nom de la communauté autochtone d'origine] m'a rendu différent. Même, aujourd'hui, je pense que je ne serais même pas là à [nom d'une ville], je serais à [nom d'une communauté autochtone] en train de consommer. 


\title{
Les résignés : des jeunes qui banalisent leur expérience
}

\author{
« J'ai vécu là-dedans comme tout le monde. »
}

\section{Le placement : une distance avec la famille immédiate et en proximité avec la famille élargie}

Le parcours de prise en charge des résignés se démarque des autres par une absence de liens significatifs avec les membres de la famille immédiate et par des contacts continus avec des membres de la famille élargie. La fratrie est très importante aux yeux des résignés, mais ils ne partagent pas une grande proximité avec leurs frères et sœurs pendant leur placement, souvent parce que ces derniers sont placés dans des milieux différents. En ce qui concerne la mère, les jeunes de la figure des résignés vivent, tout au long de leur prise en charge, une distance physique et relationnelle avec elle. Les jeunes de la figure des résignés sont confrontés à un certain désengagement de la part de leur mère, ce qui leur fait ressentir de la rancune à son égard. Toutefois, contrairement aux résistants, ces jeunes ne cherchent pas à établir un lien différent avec leur mère pendant la prise en charge et ils misent plutôt sur leurs rapports avec les membres de la famille élargie. La famille élargie est particulièrement significative pour les jeunes de la figure des résignés et elle occupe une place fondamentale dans le parcours de ces jeunes, soit parce qu'ils sont placés chez des tantes, oncles ou grands-parents, soit parce que ces derniers s'investissent grandement auprès d'eux et qu'ils les côtoient continuellement :

\section{Mère}

Je n'irais pas dire des grosses histoires à ma mère. On se parle comme ça.

C'est pour ça justement [parce que je me suis fait agresser par mon beau-père], je ne suis pas proche de ma mère. Je la vois deux fois par an. [...] On n'est pas proches pantoute. C'est sûr que c'est ma mère. Mais je vois qu'elle a pris mon beau-père à la place de ses enfants.

\section{Fratrie}

[...] La plus jeune [sœur], le bébé de la famille, je ne sais même pas où est-ce qu'elle est rendue. Je pense qu'elle est rendue à [nom d'une autre ville]. Je ne sais pas elle est où. Je l'ai vue, elle avait 3 ans. Aujourd'hui je pense qu'elle a 7 ans.

\section{Famille élargie}

Je suis parti. [...] J'ai demandé d'aller habiter avec ma grand-mère. [...] Ç’a été un placement plus tard. Comme si c'était ma grand-mère qui m'avait... pas adopté, mais qui aurait pris la garde. [...] [Je suis retourné dans ma famille] mais pas tout de suite, je ne suis pas retourné tout de suite.

[Ma tante] elle est ici [en ville]. [...] Oui, je suis proche d'elle. II y a du monde qui pense que c'est ma mère. Parce que le monde n'ont jamais vu ma mère donc ils pensent que c'est ma mère. 


\section{L'effet de la prise en charge : un détachement}

La grande proximité avec la famille élargie, combinée à l'absence de liens continus et significatifs avec la famille immédiate, fait que les jeunes de la figure des résignés vivent leur prise en charge comme un détachement. Avec leur placement, ces jeunes ont le sentiment de devoir renoncer à une proximité avec leur famille immédiate, ce qui brise leur idéal familial. L'implication des membres de la famille élargie auprès d'eux donne toutefois l'impression à ces jeunes que les ressources nécessaires à leur bien-être sont disponibles dans leur milieu d'origine. En effet, si la sécurité de ces jeunes et leurs besoins fondamentaux, matériels et affectifs, ne peuvent être assurés par leur famille immédiate, ils peuvent compter, en totalité ou en partie, sur la famille élargie pour y pourvoir. Cette situation comble, par la même occasion, leur besoin d'appartenance à leur milieu d'origine. Ainsi, les membres de la famille élargie occupent une place essentielle dans la vie de ces jeunes et les milieux d'accueil leur apparaissent secondaires. Dans ce contexte, les jeunes de cette figure accordent une plus grande importance à leur famille élargie, mais restent quand même ouverts aux ressources que peuvent leur procurer les milieux d'accueil dans lesquels ils pourront créer ou non des liens significatifs.

\section{Famille immédiate (détachement)}

[La chose dans mon parcours que j'aurais aimée qui se passe différemment c'est d'] être une famille unie. Ma mère et mon père avec mes frères et sœurs.

J'aurais aimé ça être proche de mes frères et sœurs. [...] Ils étaient en famille d'accueil à [nom d'une ville] et moi j'étais ici [chez ma tante].

\section{Famille élargie (appartenance)}

Je l'ai vue [ma mère] parce que j'étais allé voir mon petit frère [au centre de réadaptation] et elle était là aussi. C'était quand ça... Ah, à la fête de ma grand-mère. Puis depuis que ma sœur est décédée, on passe Noël toute la famille, tous mes cousins, cousines. Ça nous a rapprochés un peu plus, la famille. [...] C'est sûr qu'il y en a qui manquent parce qu'ils ne sont pas capables d'être là. Mais on essaie le plus possible. [...] Je suis bien content. Ça fait juste du bien d'être avec sa famille.

C'est sûr que je voulais retourner [vivre chez ma grand-mère].

\section{Famille d'accueil}

[...] ça a pris un petit bout avant qu'ils m'envoient [en famille d'accueil] parce qu'un moment donné ma tante était à boutte et c'est là qu'elle m'envoyait. [...] [Dans la famille d'accueil] j'ai habité là deux ans, je suis retourné chez ma tante. Je n'aimais pas beaucoup ça [dans la famille d'accueil]. [...] [Je n'aimais pas] la famille. Je n'étais pas proche d'eux autres. Je n'étais pas à l'aise mettons de demander quelque chose... C'est sûr que quand j'en avais vraiment besoin, je le demandais. 
Ça a pris un mois, même pas [avant que je sois bien dans ma famille d'accueil]. Lui il s'appelle [nom du père d'accueil], c'est quelqu'un de drôle. Puis [nom de la mère d'accueil], elle, elle parle beaucoup. Ils me niaisaient souvent, ça a fait que je me sentais bien.

Le parcours de prise en charge des résignés se vit avec des hauts et des bas et les événements qui surviennent sont vécus avec une certaine indifférence. S'ils ont été et sont toujours parfois confrontés à des difficultés, celles-ci ne sont pas vécues comme des embûches ou des obstacles, ni comme des occasions pour grandir : elles semblent faire partie du cours normal de la vie. En somme, les jeunes de la figure des résignés se détachent de leurs émotions liées à leur prise en charge et restent impassibles par rapport aux événements qui surviennent dans leur vie. Ces événements sont vus comme banals, faisant partie du cours naturel des choses, et ils peuvent, à l'occasion, en retirer des apprentissages positifs pour eux.

Je ne peux pas vraiment me rappeler mes émotions à moi, ça fait longtemps quand même... c'est une période [avant la prise en charge] que j'ai mise de côté, que j'ai oubliée un peu. Ça m'arrivait d'y penser, mais c'est rare.

Je ne crois pas que mon parcours de prise en charge a eu des conséquences sur ma vie. Je trouve que je m'en suis bien sorti. Parce que je n'ai pas tourné sur la drogue ou alcoolique... J'ai eu ma passe là-dedans, mais c'est ça...

Ma vie, elle ne m'a pas affectée. C'est sûr que je manque de confiance en moi, ça je l'ai tout le temps eu. [...] Je n'ai pas vraiment pensé pourquoi j'étais de même. Je suis très gênée aussi. Avant j'étais très gênée quand j'étais jeune. [...] [Je me sens encore gênée], mais pas aussi pire qu'avant.

\section{La synthèse de la figure des Résignés : une protection "banale "}

Pour les résignés, le placement à l'extérieur de leur famille représente une situation ordinaire. Les résignés perçoivent leur prise en charge comme une protection "banale " puisqu'elle leur permet de se soustraire aux difficultés vécues dans leur famille immédiate tout en restant en proximité avec leur famille élargie. Ces jeunes ne distinguent pas leur vécu de celui des autres jeunes n'ayant pas connu de placement, sinon par le fait qu'ils doivent renoncer à leur idéal familial :

Ce n'est pas des mauvais souvenirs, j'ai vécu là-dedans comme tout le monde. Comme ma blonde, je suis tout à fait différent de ma blonde. Ma blonde est proche de ses frères et sœurs. [...] c'est plus différent. C'est sûr que je vois ma famille de temps en temps, mais ce n'est pas aussi proche. [...] Elle, elle est très proche avec ses parents et son frère.

Pour les résignés, l'importance est mise sur la place de la famille élargie dans leur vie, parce que celleci leur permet d'avoir une vie "normale » malgré tout. Ainsi, lorsqu'on demande à une jeune s'il y a des choses qui ont été bonnes dans son parcours de prise en charge, celle-ci nous répond : "Le fait d'avoir habité avec ma grand-mère. " 


\section{Discussion}

Considérant le nombre limité de participants à l'étude, il est possible que les témoignages recueillis ne représentent pas l'éventail complet des points de vue des jeunes autochtones par rapport à leur expérience de prise en charge. Nous tenons à rappeler que cette étude ne se veut pas exhaustive quant aux perspectives possibles sur la prise en charge ; elle cherche plutôt à examiner en profondeur divers récits de vie pour éclairer l'expérience de prise en charge des jeunes autochtones. Notons également que les résultats ne peuvent être généralisés aux jeunes ayant choisi de vivre dans leur communauté d'origine après la fin de leur prise en charge, puisque la façon dont les jeunes perçoivent leur parcours de prise en charge peut être en lien avec le choix de s'établir ensuite en ville ou dans leur communauté d'origine. Toutefois, nous l'avons mentionné, cette étude a le mérite de donner la parole à de jeunes Autochtones résidant en milieu urbain, dont la voix est trop souvent ignorée.

Cette étude permet de comprendre, à la lumière des récits de vie des jeunes, le vécu de la prise en charge et les effets que celle-ci peut avoir sur les jeunes autochtones dans le contexte actuel de la protection de l'enfance au Québec. Les résultats montrent que le placement des jeunes autochtones par la protection de la jeunesse comporte des enjeux relativement à la continuité des liens avec leur famille d'origine. Les témoignages des jeunes rencontrés mettent en évidence que les relations qu'ils entretiennent avec leur famille d'origine pendant le placement sont primordiales à leurs yeux et influencent la perception qu'ils ont de leur prise en charge. On voit par le discours des jeunes rencontrés que la continuité des liens avec la famille d'origine pendant le placement représente un ancrage identitaire qui comble un besoin d'appartenance, ce qui les aide à s'adapter au milieu d'accueil. Le placement a l'effet d'un déracinement pour les jeunes qui n'ont pas de contact avec leur famille d'origine, alors qu'il a un effet d'émancipation pour les jeunes ayant des liens significatifs et continus avec leur famille d'origine et particulièrement avec leur famille immédiate. Et cet effet du placement a une influence sur le regard qu'ils portent rétrospectivement sur leur prise en charge, celle-ci étant ressentie comme ayant apporté une protection «partielle » ou au contraire une protection « totale ». Le schéma 1 illustre les résultats de recherche. 


\section{Schéma 1. L'expérience de prise en charge des jeunes autochtones lors d'un placement}

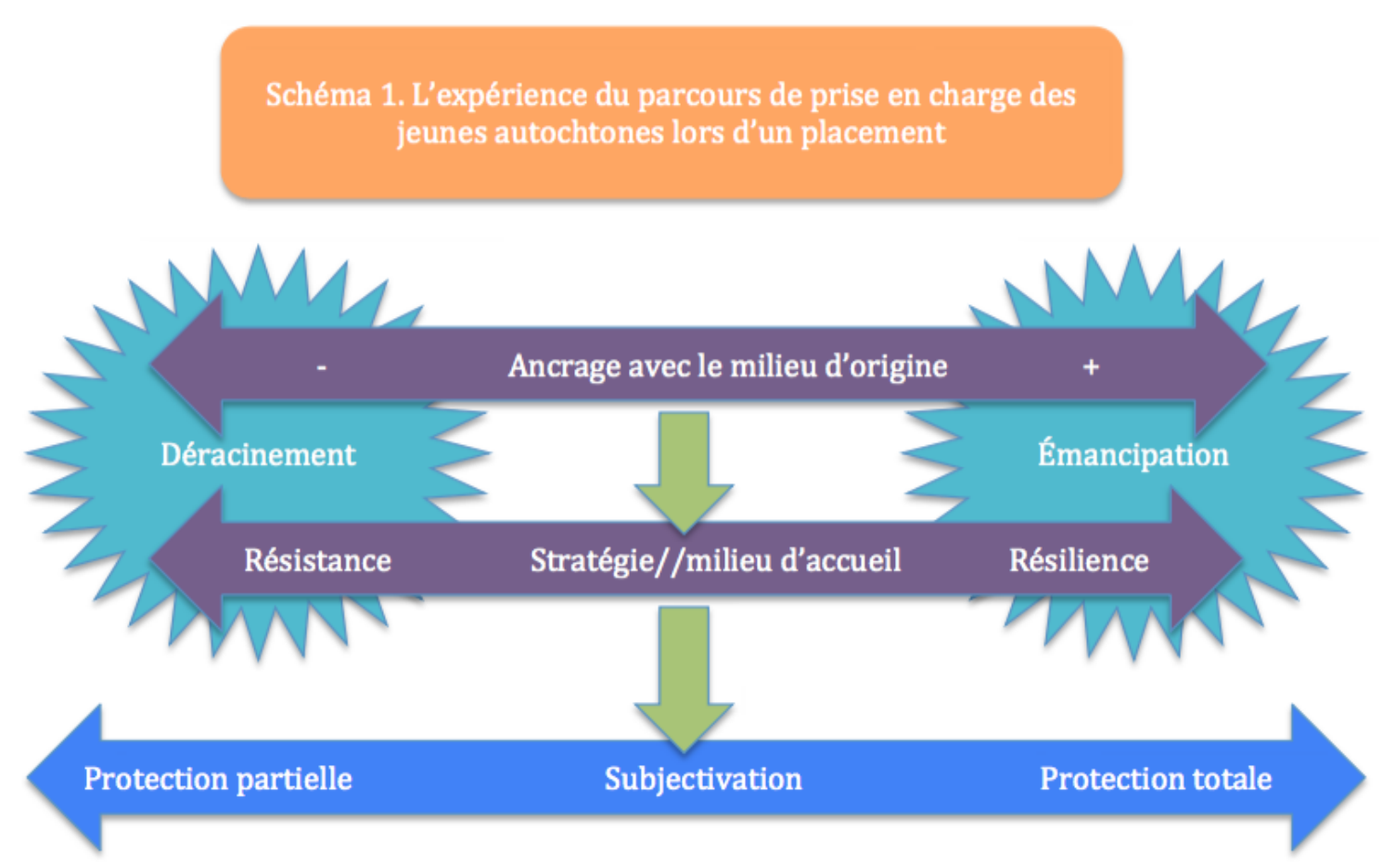

Par ailleurs, la relation avec la mère est apparue très importante pour les jeunes de notre étude, mais les relations avec la famille élargie sont aussi ressorties comme étant significatives pour ces jeunes. Les jeunes autochtones qui vivent une coupure avec leur famille immédiate mais qui entretiennent une relation significative avec leur famille élargie ne perçoivent pas la prise en charge de manière négative, à la différence de ceux qui n'ont pas de contacts avec leur famille élargie. Ces résultats pourraient s'expliquer par la conception de la famille chez les Autochtones, selon laquelle la mère n'est pas la seule contributrice au bien-être physique et émotionnel d'un enfant (Guay, 2015 ; CRPA, 1996). Dans un article sur la théorie de l'attachement, Neckoway, Brownlee et Castellan (2007) affirment que la réalité d'un enfant autochtone et ses expériences de socialisation sont différentes de celles d'un enfant non autochtone puisque le lien entre l'enfant autochtone, son parent et ses autres donneurs de soins est « multi-interactionnel » plutôt que " dyadique ». Selon nos résultats, la relation avec la mère aurait des qualités irremplaçables, mais celles entretenues avec la famille élargie seraient grandement à considérer lorsqu'on cherche à réduire les impacts négatifs du placement sur les jeunes autochtones.

D'autre part, en mettant en exergue le lien entre la proximité des jeunes autochtones avec leur milieu d'origine et l'effet du placement perçu par les jeunes, cette étude montre que les pratiques québécoises actuelles en protection de l'enfance peuvent entraîner certaines conséquences pour les jeunes autochtones qui rappellent celles qu'ont eues les pensionnats indiens et la rafle des années 1960. Selon nos résultats, l'absence de liens avec la famille élargie ajoutée à l'absence de liens avec la famille immédiate a des répercussions sur l'identité des jeunes autochtones, entraînant des conséquences 
négatives importantes dans leur vie, telles que des difficultés dans leurs rapports avec le monde extérieur et des fragilités multiples. Ces résultats font écho à d'autres recherches canadiennes, dont celle de Carrière (2008) qui porte sur les impacts de l'adoption sur l'identité des enfants autochtones et qui montre qu'une relation causale existerait entre la proximité avec la famille d'origine, la communauté et les connaissances ancestrales et la santé des jeunes autochtones adoptés.

\section{IMPLICATIONS POUR L'INTERVENTION}

Cette recherche fait ressortir certains enjeux spécifiques à l'intervention auprès des jeunes autochtones dans le contexte de la protection de la jeunesse. Lorsqu'il est impossible pour un enfant autochtone de rester dans sa famille immédiate, cette étude met en évidence qu'il s'avère essentiel d'explorer les ressources que comporte la famille élargie pour la prise en charge de l'enfant. En protection de la jeunesse, la famille élargie est en principe la première option à considérer lorsqu'il est question de placement. Cependant, les modalités des interventions peuvent être discutées quand il s'agit de familles autochtones. Sur la base de ses recherches, Guay (2010) souligne que les grands-parents autochtones acceptent souvent d'avoir la garde de leurs petits-enfants, mais que lorsqu'on leur propose l'adoption légale, ils ne sont pas d'accord et sont portés à se désinvestir. Ouellette et Goubau (2009) remarquent pour leur part que le placement à long terme ou la tutelle, qui correspondraient mieux aux réalités autochtones, semblent souvent écartés par les intervenants qui considèrent que la permanence et la stabilité ne peuvent se concrétiser qu'à travers l'adoption plénière. Puisque ces enjeux sont essentiels en contexte autochtone, les interventions devraient chercher le plus possible l'implication de la famille élargie auprès des jeunes autochtones ; cela pourrait passer par exemple par davantage de flexibilité dans les procédures, ou par la reconnaissance de l'adoption coutumière qui est une tradition déjà présente chez les populations autochtones (Gouvernement du Québec, 2012 ; Guay et Grammond, 2012).

En outre, lorsqu'il devient incontournable de placer un jeune autochtone à l'extérieur de son milieu d'origine, nos données montrent qu'il est essentiel de favoriser la continuité des liens avec sa famille immédiate et élargie. Le placement avec la fratrie et les sorties régulières dans la famille d'origine apparaissent comme des moyens à favoriser pour permettre aux jeunes de rester en lien continu avec leur milieu d'origine. On peut toutefois se demander comment il est possible de privilégier les relations avec le milieu d'origine alors qu'il ressort des témoignages des jeunes que c'est souvent un désengagement de la part de leurs proches qui est à la source de la rupture des liens. Au regard de nos données de recherche, nous pouvons faire ressortir un élément explicatif. Les jeunes que nous avons rencontrés ont rapporté que leurs parents avaient une image très négative du système de protection de la jeunesse. II est possible que cette vision négative des services de protection ne soit pas étrangère au désengagement des parents vis-à-vis de leur enfant, lequel s'expliquerait ainsi plutôt par un renoncement face à un système perçu comme colonialiste. Les familles autochtones ont été très éprouvées par les pratiques de protection qui ont régné dans le passé et on le comprend bien. Le sentiment d'échec et de honte ressenti lorsqu'on perd la garde de son enfant peut être doublement puissant quand on " se le fait enlever » par un système dominant qui n'est pas le nôtre et qui ne représente pas nos valeurs et nos conceptions familiales. Dans ce contexte, une attention portée, dans les interventions, à la perception qu'ont les familles autochtones des services qui leur sont offerts semble être une avenue intéressante. Des formations sur le thème de la sécurité culturelle pour les intervenants et familles d'accueil pourraient aider à faire baisser les tensions existantes entre le système 
de protection de la jeunesse et les familles autochtones et ainsi, souhaitons-le, contribuer à la mobilisation des membres de la famille auprès des jeunes qui ont été placés.

Les témoignages des jeunes rencontrés amènent également à se questionner sur le rôle que jouent les intervenants en protection de la jeunesse. II apparaît dans le discours des jeunes autochtones que la présence de leurs intervenants, sauf exception, n'a pas été significative dans leur vie. La famille d'origine et les familles d'accueil occupent une place beaucoup plus importante en ce qui a trait à leur bien-être. Comme le lien des jeunes avec leur famille d'origine est primordial pour eux, ne serait-il pas pertinent pour les intervenants de miser sur cette relation en jouant un rôle d'accompagnateur de la famille plutôt que de centrer les interventions sur les jeunes ? Cela répondrait davantage à la vision autochtone qui privilégie l'intervention familiale plutôt que l'intervention individuelle (Femmes autochtones du Québec [FAQ] et Regroupement des centres d'amitié autochtone du Québec [RCAAQ], 2005). En ce sens, des pratiques d'intervention telles que les cercles de famille (Desmeules, 2007), par lesquels les membres de la famille participent aux décisions concernant l'enfant et qui favorisent la mobilisation des membres de la famille autour de l'enfant, seraient à privilégier. D'autre part, cette étude fait ressortir que certains comportements qui sont source de conflits avec la famille d'accueil ainsi que les fugues peuvent être des manières, pour les jeunes autochtones, d'exprimer un malaise identitaire. À ce titre, les interventions punitives, comme la restriction des sorties dans la famille d'origine, ne seraient pas bénéfiques pour ces jeunes. Bien au contraire, elles pourraient amplifier la raison même de ces comportements. Les intervenants auraient donc tout intérêt à être sensibles à ce que veulent nous dire les jeunes autochtones en adoptant ce type de comportements et devraient adopter une approche compréhensive plutôt que punitive quand il est question de contacts avec leur famille.

À la lumière des enjeux qui ressortent de cette étude, la gouvernance des services de protection de la jeunesse par les communautés autochtones elles-mêmes apparaît comme une solution bénéfique pour les jeunes autochtones qui nécessitent une prise en charge. Les populations autochtones considèrent l'intérêt de l'enfant inséparable de l'intérêt de sa famille, de sa communauté et de sa culture (Richard, 2004 ; Kline, 1992). En redonnant aux populations autochtones leur autonomie décisionnelle en matière de protection de la jeunesse, on peut penser que les décisions que celles-ci prendront refléteront inévitablement leur vision de l'intérêt de l'enfant, et que les solutions mises en place correspondront mieux aux besoins des enfants autochtones. En outre, dans un contexte où les communautés autochtones ont la responsabilité des services de protection de la jeunesse, les chances sont plus grandes que les interventions puissent mobiliser la famille immédiate et élargie autour de l'enfant, puisque celles-ci sont porteuses des mêmes valeurs et de la même culture. Les cercles de famille qui sont inspirés de pratiques ancestrales autochtones en sont un bel exemple (Desmeules, 2007). Après avoir questionné des intervenants autochtones, Guay (2010) fait d'ailleurs le constat que ce qui pose problème pour eux n'est pas tant le fondement de la LPJ mais la manière de l'appliquer dans les communautés autochtones. Finalement, puisque cette étude montre que le placement des jeunes autochtones par les services sociaux comporte des enjeux identitaires pour ces jeunes, il importe d'agir en amont de la prise en charge si l'on veut réellement considérer leur intérêt avant tout. Les populations autochtones doivent pouvoir bénéficier des mêmes conditions de vie que le reste de la population canadienne si l'on veut que les jeunes autochtones puissent avoir la chance de grandir et s'épanouir positivement dans leurs familles et leurs communautés. À cet égard, un financement doit être accordé en fonction des besoins réels des communautés et ces dernières doivent pouvoir bénéficier d'une 
latitude pour développer des solutions spécifiques aux problématiques auxquelles elles font face. La surreprésentation des jeunes autochtones en protection de la jeunesse prend racine dans la colonisation et les solutions doivent donc, à tous égards, être innovantes pour tenir compte de ce contexte. Cela rejoint d'ailleurs les appels à l'action de la Commission de vérité et de réconciliation (CVR, 2015).

\section{Conclusion}

Les populations autochtones du Canada ont un passé marqué par la colonisation et les politiques assimilatrices qui affecte encore aujourd'hui la réalité des jeunes autochtones. La surreprésentation de ceux-ci dans les services de protection de l'enfance en est bien la preuve. Si des recherches ont permis de faire la lumière sur les causes de cette surreprésentation, cette étude est, à notre connaissance, la première au Canada à s'intéresser au point de vue des jeunes autochtones sur leur expérience de prise en charge. En explorant les perceptions des jeunes autochtones sur la façon dont ils avaient vécu celleci, il a été possible de dégager trois types d'expérience. Il en est ressorti que pour favoriser le bien-être des jeunes autochtones en situation de placement, on doit leur permettre de conserver le plus grand ancrage possible dans leur famille d'origine et préserver les liens familiaux. Selon nos résultats, les jeunes qui bénéficient de cet ancrage identitaire s'adaptent plus facilement au milieu d'accueil et adoptent des stratégies positives dans leurs relations avec le monde extérieur. À l'opposé, les jeunes autochtones qui ne bénéficient pas de cet ancrage identitaire expriment de la difficulté à s'adapter à leur milieu d'accueil et adoptent des stratégies de résistance dans leurs relations avec le monde extérieur. L'expérience de ces derniers rappelle les impacts des pensionnats et de la rafle des années 1960, ce qui démontre que des efforts considérables doivent être faits si l'on veut faire une coupure avec les pratiques du passé. Ces résultats invitent à faire plus de place à la vision autochtone de l'intérêt de l'enfant, où celui-ci est inséparable de l'intérêt de sa famille, de sa communauté et de sa culture. Des pistes concernant la pratique ont été discutées, mais ultimement, la gouvernance des services de protection de la jeunesse par les communautés autochtones elles-mêmes semble l'avenue à privilégier. Finalement, du point de vue théorique, puisque cette étude a été réalisée auprès de jeunes autochtones résidant en milieu urbain, il serait pertinent lors de futures recherches de recueillir le point de vue de jeunes autochtones ayant choisi de s'établir dans leur communauté d'origine à la fin de leur prise en charge, afin de compléter la typologie proposée dans cet article. 


\section{RÉFÉRENCE}

Agence de la santé publique du Canada (2013). Les jeunes autochtones : le pouvoir guérisseur de l'identité culturelle. [http://www.phac-aspc.gc.ca/hp-ps/dca-dea/prog-ini/ahsunc-papacun/aboriginalautochtones-fra.php]

Armitage, A. (1995). Comparing the policy of Aboriginal assimilation: Australia, Canada and New Zealand, Vancouver, University of British Columbia Press.

Atwool, N. (2006). «Participation in decision-making: The experience of New Zealand children in care », Child Care in Practice, vol. 12, n 3, p. 259-267.

Bennett, M., et Blackstock, C. (2002). Recensement des écrits et bibliographie annotée traitant de certains aspects du bien-être des enfants autochtones au Canada, Ottawa, Centre d'excellence pour la protection et le bien-être des enfants.

Bennett, M., Blackstock, C., et De La Ronde, R. (2005). A literature review and annotated bibliography on aspects of Aboriginal child welfare in Canada, Ottawa, First Nations Child and Family Caring Society of Canada.

Bertaux, D. (2010). L'enquête et ses méthodes. Le récit de vie, Paris, Armand Colin.

Blackstock, C. (2009). "The occasional evil of angels: Learning from the experiences of Aboriginal peoples and social work », First Peoples Child and Family Review, vol. 4, n 1, p. 28-37.

Blackstock, C. (2011). "Why if Canada wins, Canadians lose: The Canadian Human Rights Tribunal on First Nations Child Welfare », Children and Youth Services Review, vol. 33, p. 187-194.

Blackstock, C., et Trocmé, N. (2005). "Community-based child welfare for Aboriginal children: Supporting resilience through structural change ", Social Policy Journal of New Zealand, vol. 24, p. $12-33$.

Blackstock, C., Trocmé, N., et Bennett, M. (2004). « Child maltreatment investigations among Aboriginal and non-Aboriginal families in Canada: A comparative analysis ", Violence Against Women, vol. 10, $\mathrm{n}^{\circ} 8$, p. 901-916.

Carrière, J. (2008). "Maintaining identities: The soul work of adoption and Aboriginal children », $A$ Journal of Aboriginal and Indigenous Health Community, vol. 6, n ${ }^{\circ}$, p. 61-80.

Castellano, M. B. (2002). Aboriginal family trends: Extended families, nuclear families, families of the heart, Ottawa, Vanier Institute of the Family.

Castellano, M. B., Archibald, L., et DeGagné, M. (2008). De la vérité à la réconciliation. Transformer l'héritage des pensionnats, Ottawa, Fondation autochtone de guérison.

Commission de vérité et réconciliation du Canada (CVR) (2015). Honorer la vérité, réconcilier pour l'avenir. Sommaire du rapport final de la Commission de vérité et réconciliation du Canada. 
[http://www.trc.ca/websites/trcinstitution/File/French_Exec_Summary_web_revised.pdf]

Commission royale sur les peuples autochtones (CRPA) (1996). Rapport de la Commission royale sur les peuples autochtones, rapport final, Ottawa, Gouvernement du Canada.

Daunais, J.-P. (1995). «L'entretien non directif », dans B. Gauthier (dir.), Recherche sociale. De la problématique à la collecte des données, Sainte-Foy, Presses de l'Université du Québec, p. 273293.

Demers, L. (2009). « Le système de protection au Québec : I'organisation des services en soutien aux enfants en difficulté et à leur famille ", Santé, société et solidarité, vol. 1, p. 81-89.

Desmeules, G. (2007). «A sacred family circle: A family group conferencing model », dans I. Brown, F. Chaze, D. Fuchs, J. Lafrance, S. McKay et S. Thomas Prokop (dir.), Putting a human face on child welfare: Voices from the Prairies, Regina/Montréal, Prairie Child Welfare Consortium/Centre of Excellence for Child Welfare, p. 161-188.

Dion Stout, M., et Kipling, G. (2003). Peuples autochtones, résilience et séquelles du régime des pensionnats, Ottawa, Fondation autochtone de guérison.

Dubet, F. (1994). La sociologie de l'expérience, Paris, Éditions du Seuil.

Femmes autochtones du Québec (FAQ) et Regroupement des centres d'amitié autochtone du Québec (RCAAQ) (2005). Mémoire conjoint concernant la révision de la Loi sur la protection de la jeunesse. [http://www.faq-qnw.org/documents/memoirelpj]

Fournier, S., et Crey, E. (1997). Stolen from our embrace: The abduction of First Nations children and the restoration of Aboriginal communities, Vancouver, Douglas and McIntyre.

Gagnon Dion, M.-H., Rivard, J., Bellot, C. (2017). « Jeunes autochtones et protection de la jeunesse : leur point de vue sur leur prise en charge", Sociétés et jeunesses en difficulté. Revue pluridisciplinaire de recherche, $\mathrm{n}^{\circ}$ 19. [https://journals.openedition.org/sejed/8507]

Gouvernement du Québec (2012). Rapport du Groupe de travail sur l'adoption coutumière en milieu autochtone, Québec, Ministère de la Justice du Québec.

Guay, C. (2010). La rencontre des savoirs à Uashat mak Mani Utenam : regards des intervenants sociaux sur leur pratique, thèse de doctorat, Université du Québec en Outaouais.

Guay, C. (2015). "Les familles autochtones : des réalités sociohistoriques et contemporaines aux pratiques éducatives singulières ", Intervention, $\mathrm{n}^{\circ} 141$, p. 17-27.

Guay, C., et Grammond, S. (2012). "Les enjeux de l'application des régimes de protection de la jeunesse aux familles autochtones », Nouvelles pratiques sociales, vol. 24, nº 2, p. 67-83.

Kline, M. (1992). " Child welfare law, "best interests of the child" ideology, and First Nations ", Osgoode Hall Law Journal, vol. 30, n², p. 375-425. 
Little Bear, L. (2000). « Jagged worldviews colliding », dans M. Battiste (dir.), Reclaiming

Indigenous voice and vision, Vancouver, University of British Columbia Press, p. 77-85.

McKenzie, H. A., Varcoe, C., Browne, A. J., et Day, L. (2016). «Disrupting the continuities among residential schools, the sixties scoop, and child welfare: An analysis of colonial and neocolonial discourses », International Indigenous Policy Journal, vol. 7, $\mathrm{n}^{\circ} 2$.

Neckoway, R., Brownlee, K., et Castellan, B. (2007). "Is attachment theory consistent with aboriginal parenting realities? », First Peoples Child and Family Review, vol. 3, n 2, p. 65-74.

Ouellette, F.-R., et Goubau, D. (2009). « Entre abandon et captation. L'adoption québécoise en "banque mixte" », Anthropologie et Sociétés, vol. 33, nº1, p. 65-81.

Richard, K. (2004). «A commentary against Aboriginal to non-Aboriginal adoption », First Peoples Child and Family Review, vol. 1, n 1, p. 101-109.

Schnapper, D. (2005). La compréhension sociologique. Démarche de l'analyse typologique, Paris, Presses universitaires de France.

Sigouin, E. (2006). Les mécanismes de protection de la jeunesse autochtone au regard de la théorie libérale de Will Kymlicka, mémoire de maîtrise, Université de Montréal.

Sinclair, R. (2007). "Identity lost and found: Lessons from the sixties scoop ", First Peoples Child and Family Review, vol. 3, n 1, p. 65-82.

Sinha, V., Fast, E., Trocmé, N., Fallon, B., et MacLaurin, B. (2010). « La composante Premières Nations de l'Étude canadienne sur l'incidence des signalements de cas de violence et de négligence envers les enfants : une approche axée sur le renforcement des capacités dans le cadre d'une recherche nationale appliquée aux Premières Nations ", Nouvelles pratiques sociales, vol. 23, n 1, p. 83-98.

Sinha, V., Trocmé, N., Fallon, B., MacLaurin, B., Fast, E., Thomas Prokop, S. ... et Richard, K. (2011). Kiskisik Awasisak: Remember the children. Understanding the overrepresentation of First Nations children in the child welfare system, Ottawa, Assembly of First Nations.

Teichroeb, R. (1997). Flowers on my grave: How an Ojibway boy's death helped break the silence on child abuse, Toronto, Harper Collins Publishers.

Tourigny, M., Domond, P., Trocmé, N., Sioui, B., et Baril, K. (2007). «Les mauvais traitements envers les enfants autochtones signalés à la Protection de la jeunesse du Québec: comparaison interculturelle », First Peoples Child and Family Review, vol. 3, n 3, p. 84-102.

Trocmé, N., Knoke, D., et Blackstock, C. (2004). «Pathways to the overrepresentation of Aboriginal children in Canada's child welfare system ", Social Service Review, vol. 78, n 4, décembre, p. 577600. 